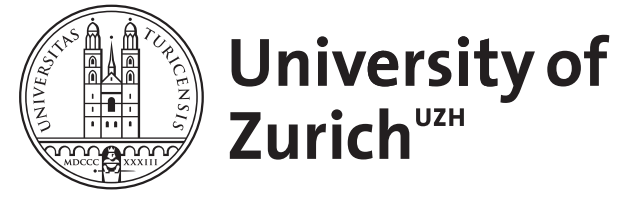

Zurich Open Repository and Archive

University of Zurich

University Library

Strickhofstrasse 39

CH-8057 Zurich

www.zora.uzh.ch

Year: 2017

\title{
Ernährung in der Schwangerschaft
}

Abt, Sibylle

Posted at the Zurich Open Repository and Archive, University of Zurich

ZORA URL: https://doi.org/10.5167/uzh-143994

Journal Article

Published Version

Originally published at:

Abt, Sibylle (2017). Ernährung in der Schwangerschaft. Schweizer Zeitschrift für Ernährungsmedizin (SZE), (3/2017):23-25. 


\title{
Ernährung während der Schwangerschaft
}

\author{
Erfahrungen aus dem Projekt PEBS/buggyfit
}

\begin{abstract}
Sibylle Abt
Eine ausgewogene Ernährung und regelmässige Bewegung sind während der Schwangerschaft wichtig. Im Projekt PEBS/buggyfit können Frauen während der Schwangerschaft und nach der Geburt Fragen zur Ernährung in einer persönlichen Ernährungsberatung klären und sich in spezifischen Fitnesskursen gemeinsam bewegen. Angesprochen sind insbesondere auch Frauen mit Migrationshintergrund.
\end{abstract}

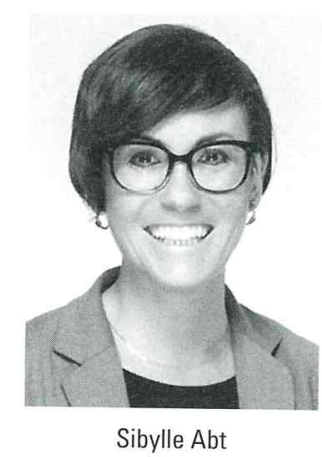

Das Projekt PEBS/buggyfit (Präventive Ernährungsund Bewegungsberatung in der Schwangerschaft bis ein Jahr nach Geburt) wurde 2009 an der Klinik für Geburtshilfe am Universitätsspital Zürich ins Leben gerufen und wird seit 2014 vom Verein PEBS geführt. Das Ziel ist die Sensibilisierung der Eltern für eine gesunde Ernährung und genügend Bewegung. Die Forschung bestätigte in den letzten Jahren die Tatsache, dass während der Schwangerschaft zentrale metabolische Regelkreise beim Kind geprägt werden, welche wegweisend für das spätere Leben sind (1). Darum können schon während der Schwangerschaft Kinder präventiv vor einer übermässigen Gewichtszunahme bewahrt und dadurch optimale Voraussetzungen für das spätere Leben geschaffen werden.

Im Fokus von PEBS/buggyfit stehen junge Frauen, welche für ihre Kinder in Bezug auf gesundes Essen und einen aktiven, sportlichen Lebensstil ein Vorbild sein wollen. Besonders sollen auch Frauen mit Migrationshintergrund für diese Angebote gewonnen werden.

Was in Zürich als Pilotprojekt startete, ist unterdessen ein etabliertes Angebot, das auch in anderen Kantonen Fuss gefasst hat. In Zürich stehen die Ernährungsberatung und die Fitnesskurse allen schwangeren Frauen in der Stadt und der Region offen.

\section{Gewichtszunahme während der Schwangerschaft}

Eine Gewichtszunahme in der Schwangerschaft ist in einem physiologischen Rahmen normal. Je nach Body-Mass-Index (BMI) vor der Schwangerschaft gelten unterschiedliche Empfehlungen bezüglich der Gewichtszunahme während der Schwangerschaft ( $\mathrm{Ta}$ belle) (2).

\section{Energie und Makronährstoffbedarf}

Während der Schwangerschaft gilt als Basis eine ausgewogene Ernährung gemäss der Lebensmittelpyramide der Schweizerischen Gesellschaft für Ernährung (SGE) mit täglich 1,5 bis 2 Liter ungesüssten Getränken und 5 Portionen Gemüse, Salat und Früchten, bunt gemischt.

Der Energiebedarf während der Schwangerschaft steigt erst ab dem 2. Trimenon auf zirka $2200 \mathrm{kcal}$ pro Tag an und erreicht im 3. Trimenon zirka $2500 \mathrm{kcal}$ pro Tag. Dies gilt für normalgewichtige Frauen (3).

Die Energiezufuhr wird folgendermassen verteilt:

- 45 bis 55 Prozent als Kohlenhydrate (200-250 g/Tag)

- 30 bis 35 Prozent Fett (80-90 g/Tag)

- 8 bis 10 Prozent Protein, wobei der Anteil an Proteinen ab dem 4. Monat ansteigt (60-70g/Tag).

Um bereits am Anfang der Schwangerschaft Ernährungsfehler und Unsicherheiten der Frauen zu klären, steht die präventive Ernährungsberatung von PEBS/ buggyfit allen Frauen ab Beginn der Schwangerschaft offen. Sinnvoll ist ein erstes Gespräch in der 10. bis 15. Schwangerschaftswoche sowie eine Reevaluation in der 26. Schwangerschaftswoche.

Es wurden bereits über 2000 Frauen allein in Zürich anhand einer persönlichen Ernährungsanamnese zu ihrem Essverhalten befragt, beraten und systematisch in einem eigens dafür entwickelten Ernährungstool erfasst.

Über 60 Prozent der Frauen haben einen Migrationshintergrund, wobei die Frauen aus rund 81 verschiedenen Ländern kamen. Die Beratung wurde in verschiede-
Lalimentation des femmes enceintes issues de I'immigration

Mots clés: Conseil nutritionnel préventif - outil pour l'alimentation - information spécifique

Le projet PEBS/buggyfit permet à toutes les femmes enceintes d'obtenir, par l'intermédiaire d'un conseil nutritionnel personnalisé, des informations et des explications sur les questions ayant trait à l'alimentation pendant la grossesse et après l'accouchement, et de pratiquer une activité physique en commun lors de cours de fitness spécifiques. Le programme PEBS/buggyfit a débuté en 2009 à Zurich et il est depuis présent dans plusieurs autres cantons. 
nen Sprachen geführt, und oftmals wurden auch Angehörige für die Übersetzung mitgenommen. Erschwerten trotzdem Verständnisprobleme die Beratung, wurde zusätzlich visuell gearbeitet und spezifisches Übersetzungsmaterial mitgegeben.

In der Auswertung des Ernährungstools zeigte sich deutlich, dass nur zirka 22 Prozent der Schwangeren die empfohlenen 4 Portionen Proteine (1 Portion Fleisch, Geflügel, Fisch, Eier, Tofu/Quorn und 3 Portionen Milch und Milchprodukte) pro Tag umgesetzt hatten. Dabei schnitten Frauen aus den asiatischen und afrikanischen Ländern am besten ab. Diese Zahlen sind allerdings nicht statistisch signifikant und müssen mit Vorsicht interpretiert werden.

Meist führte Unsicherheit oder Übelkeit bei den verbliebenen zwei Dritteln der Schwangeren dazu, dass sie viele Proteine aus ihrem Ernährungsplan strichen. Selten wurde bewusst darauf verzichtet. Durch eine spezifische Aufklärung wurde diesen Frauen in der Beratung die Bedeutung von Proteinen erklärt.

\section{Mikronährstoffe}

Trotz einer ausgewogenen Ernährung kann der Bedarf an Folsäure, Vitamin D und bei vielen Frauen auch an Eisen und Vitamin $B_{12}$ nicht über das Essen gedeckt werden.
Trotz einer ausgewogenen Ernährung kann der Bedarf an Folsäure, Vitamin D und bei vielen Frauen auch an Eisen und Vitamin $B_{12}$ nicht über das Essen gedeckt werden.

Folsäure spielt eine zentrale Rolle bei Zellteilungsprozessen und in der Blutbildung. Für Folsäure gilt die Empfehlung, bereits perikonzeptionell täglich $400 \mathrm{Mi}$ krogramm zu supplementieren und dies bis mindestens zur 12. Schwangerschaftswoche fortzuführen. Die Folsäuresubstitution vermindert das Risiko für einen Neuralrohrdefekt wie die Spina bifida (4). Zusätzlich sollten täglich folatreiche Lebensmittel (frisches Gemüse und Früchte, Vollkornprodukte) konsumiert werden.

Vitamin D gehört zu den fettlöslichen Vitaminen und ist essenziell für die Skelettentwicklung und die Knochengesundheit. Es wird sowohl über die Ernährung als auch durch UV-B-Lichtexposition zugeführt. Während der Schwangerschaft wird eine tägliche Supplementation von 600 IE (15 mg) empfohlen, da die

\section{Tabelle: \\ Empfohlene Gewichtszunahme während der Schwangerschaft in Abhängigkeit vom Body-Mass-Index (BMI)}

\begin{tabular}{l|l|l|l} 
& $\begin{array}{l}\text { BMI vor } \\
\text { Schwangerschaft } \\
\text { in } \mathrm{kg} / \mathrm{m}^{2}\end{array}$ & $\begin{array}{l}\text { Empfohlene } \\
\text { Gewichtszunahme } \\
\text { gesamt in } \mathrm{kg}\end{array}$ & $\begin{array}{l}\text { Empfohlene } \\
\text { Gewichtszunahme } \\
\text { pro Woche in } \mathrm{kg}\end{array}$ \\
\hline Normalgewicht & $18,5-24,9$ & $11,5-16$ & 0,4 ab 12. SSW \\
\hline Untergewicht & $<18,5$ & $12,5-18$ & 0,5 ab 12. SSW \\
\hline Übergewicht & $25-29,9$ & $7-11,5$ & 0,3 ab 12. SSW \\
\hline Adipositas & $\geq 30$ & $5-9$ & 0,2 ab 12. SSW \\
\hline $\begin{array}{l}\text { Zwillings- } \\
\text { schwangerschaft }\end{array}$ & $18,5-24,9$ & $17-24,5$ & \\
& $25-29,9$ & $14-22,5$ & \\
\hline SSW: Schwangerschaftswoche & $11,5-19$ & \\
\hline
\end{tabular}

tägliche Bedarfsdeckung über die Ernährung und die Sonnenexposition häufig nicht ausreicht. Ein Mangel wird als 25-Hydroxy-Vitamin D unter einem Wert von $50 \mathrm{nmol} / \mathrm{l}(20 \mathrm{ng} / \mathrm{ml})$ definiert (5). Es wird davon ausgegangen, dass in der Schweiz fast die Hälfte der Bevölkerung an einem Vitamin-D-Mangel leidet, vor allem in den Wintermonaten (6). Während der Schwangerschaft kann ein Vitamin-D-Mangel eine der Ursachen für Mangelgeburten beziehungsweise Hypotrophie bei Geburt sein (7). Der positive Effekt einer Vitamin-D-Supplementation wurde vor allem für die Knochengesundheit gezeigt. Für die Sicherheit einer Vitamin-D-Supplementation in der Schwangerschaft liegen gute Daten vor. Bei täglich bis zu 4000 IE Vitamin D pro Tag sind keinerlei unerwünschte Nebenwirkungen aufgetreten (8). Schwangere mit einem zusätzlichen Risikofaktor wie dunkler Hautfarbe, Gestationsdiabetes, BMI > 30 oder limitierter Sonnenexposition wird eine Bestimmung von 25-Hydroxy-Vitamin D im Serum empfohlen. Da Vitamin D die Kalziumaufnahme im Darm und den Einbau in die Knochen fördert, werden zusätzlich täglich 1000 mg Kalzium (3-4 Portionen Milch und Milchprodukte) empfohlen. Daher ist es umso wichtiger, dass die schwangeren Frauen diesbezüglich umfassend informiert werden und individuelle Tipps zur Umsetzung erhalten.

Eisen: Während der Schwangerschaft werden grosse Mengen an Eisen für die Blutbildung benötigt.

Bis zu 32 Prozent aller Schwangeren in der Schweiz haben einen Eisenmangel und bis zu 7 Prozent eine Eisenmangelanämie. Mütterliche Anämie ist je nach Schweregrad ein Risikofaktor für eine erhöhte Frühgeburtsrate, weshalb allen Schwangeren zur Prophylaxe einer Anämie und/oder eines Eisenmangels eine Hämoglobin- und Ferritinbestimmung zu Beginn der Schwangerschaft empfohlen wird, um frühzeitig handeln zu können (9). Mit Eisenquellen wie Fleisch und Eiern, aber auch pflanzlichen Lebensmitteln wie Vollkornprodukten, Hülsenfrüchten, Nüssen und einigen Gemüsesorten (z.B. Spinat, Krautstiele) kann die Aufnahme unterstützt werden.

Vitamin $B_{12}$ spielt eine Rolle bei der Zellteilung und der Blutbildung. Täglich werden 3,5 Mikrogramm empfohlen. Da es vor allem in tierischen Lebensmitteln vorkommt, sollten Vegetarierinnen und Veganerinnen dieses Vitamin supplementieren (10).

Fettsäuren: Ferner ist zu beachten, dass die Ernährung reich an mehrfach ungesättigten Fettsäuren ist. Die langkettigen, mehrfach ungesättigten Fettsäuren (Long Chain Polyunsaturated Fatty Acids, LCPUFA) sind essenziell. Aus Alpha-Linolensäure baut der Organismus langkettige Fettsäuren wie die Docosahexaensäure (DHA) auf. Diese sind zentral für die Gehirnentwicklung und die visuelle und kognitive Entwicklung des Fetus. Es sollten täglich Omega-3Fettsäuren in Form von mindestens 200 mg DHA aufgenommen werden (11). Dies kann mit 1 bis 2 Portionen Fisch pro Woche, vor allem fettreichem Fisch, oder mit Fischölkapseln erreicht werden. Keinen Einfluss hat die Supplementation von Omega-3-Fettsäu- 
ren in Form von Fischölkapseln auf die Raten von Präeklampsie, Frühgeburten, tiefem Geburtsgewicht und auf Kinder mit Small-for-Gestational-Age (12). Jod wirkt als Bestandteil der Schilddrüsenhormone Trijodthyronin (T3) und Thyroxin (T4). Es ist für ein normales Wachstum und die Entwicklung wesentlich. Der Bedarf steigt während der Schwangerschaft auf 250 Mikrogramm Jod pro Tag an. Der Anteil an Jod ist in den meisten Lebensmitteln gering, deshalb wird jodiertes Salz zum Kochen empfohlen. Entgegen den Empfehlungen in Deutschland wird in der Schweiz die Supplementation von Jod in der Schwangerschaft nicht forciert. Aufgrund kontinuierlicher Abnahme der Jodversorgung bei schwangeren Frauen (2004: mittlere Jodkonzentration im Urin [mUIC] = 249 ug/l; 2009: mUIC = 162 ug/l) (13) wurde die Jodkonzentration im Kochsalz im Jahr 2014 von 20 ppm auf 25 ppm erhöht (14). Neue Daten über die Jodversorgung schwangerer Frauen werden dieses Jahr erwartet.

\section{PEBS/buggyfit}

Das Projekt PEBS/buggyfit ist in der Schweiz einzigartig, weil es Ernährung, Fitness und Bewegung an den meisten Standorten kombiniert anbietet. Das erleichtert und fördert die Prävention. Durch die spezifische Aufklärung mit Übersetzern und visuellen Kommunikationssystemen und spezifischem Übersetzungsmaterial ist PEBS/buggyfit auch ein niederschwelliges Angebot für Frauen mit Migrationshintergrund.

\section{Korrespondenzadresse:}

Sibylle Abt

MAS Prevention and Health Promotion

Dipl. Ernährungsberaterin Bsc

Geschäftsführerin Verein PEBS

Unversitätsspital Zürich

Frauenklinikstrasse 10

8091 Zürich

E-Mail: sibylle.abt@usz.ch

Internet: www.buggyfit.ch

Literatur auf www.sze.ch abrufbar

\section{Mit Andreafol ${ }^{\circledR}$ beugen Sie Neuralrohrdefekten vor!}

\section{Das BAG empfiehlt: «Folsäure-Supplementierung von $\mathbf{0 . 4} \mathbf{~ m g ~} \mathbf{4}$ Wochen präkonzeptionell}

bis zur 12. SSW, evtl. ab der 12. SSW Vitamin-Supplementierung.»*



